\title{
Bistable Flow Driven by Coloured Gaussian Noise: A Critical Study
}

\author{
P. Hänggi ${ }^{1}$, F. Marchesoni ${ }^{2}$, and P. Grigolini ${ }^{1}$ \\ ${ }^{1}$ Dipartimento di Fisica, Università di Pisa, and GNSM del CNR, Pisa, Italy \\ ${ }^{2}$ Dublin Institute for Advanced Studies, Dublin, Ireland
}

Received January 30, 1984

\begin{abstract}
A one-dimensional bistable flow driven by additive, exponentially correlated Gaussian noise is considered. The small relaxation time Fokker-Planck approximations, widely used in the recent literature, are derived and possible shortcomings of those approximation schemes are discussed. In particular, it is pointed out that higher order nonFokker-Planck type contributions are generally of the same order as the Fokker-Planck terms. In principle, those contributions cannot be neglected if the global behavior of the probability solutions is to be described accurately. The result for the activation rate (Arrhenius factor), as evaluated from the approximative Fokker-Planck schemes, does not coincide in leading order in the correlation time $\tau$ of the noise with a computer simulation of the rate at low noise level. This result indicates that the wings of the stationary probability $\bar{p}(x)$ are in leading order in $\tau$ not recovered correctly from the approximative Fokker-Planck schemes. Some implications of our study for adiabatic elimination procedures are also discussed.
\end{abstract}

\section{Introduction}

There has been recent interest in non-linear systems subjected to external noise with a finite correlation time. In many situations the influence of a finite correlation time $\tau$ on the dynamics of a macroscopic variable plays a minor role such that an approximative Markovian theory, e.g. a Fokker-Planck description, modelling the statistical macroscopic flow, is justified $[1,2]$. On the other hand there exist cases where the influence of the bath on the macroscopic flow of an order parameter must be modelled with a coloured noise source [3-6]. A well-known example of this kind of situation is the phenomenon of motional narrowing in magnetic resonance. Kubo $[4,7]$ has shown that a very short correlation time of the fluctuating magnetic field yields a vanishing effect on the motion of the spin; on the contrary, if the fluctuations of the field are large and correlated over a long time scale, the motion of the spin is greately modified. Another important example is the relevant influence of the correlated noise on the activation rates in equilibrium systems [8-11] and in driven non-equilibrium systems [12].

Generally, the finite correlation of the noise will have an effect on the form of the stationary probability. This fact has been exploited in recent studies of so-called colored noise induced transitions [1318]. Because the underlying dynamics are governed by a non-Markovian process, the exact master equation can be obtained in special cases only $[5,12-14$, $18,19]$. Therefore, one generally must invoke an approximation procedure such as the small relaxation time Fokker-Planck schemes put forward by the advocates of $[14,15,20-22]$. Moreover, it has been pointed out previously on several occasions [1, 5, 23-25], that with a non-Markovian dynamics, the initial preparation procedure, being reflected in the statistical properties of the correlated noise [25], is of equal importance as the macro-dynamical law generated from an initial probability $p_{0}(x)$ of the macrovariables $x(t)$. Therefore, caution must be exercised in interpreting correctly the statistical information of the quantities calculated by means of a generalized Langevin equation or master equation dynamics $[5,25,26]$.

In Sect. 2, we present the model of an overdamped particle motion in a symmetric double-well potential 
being driven by an exponentially correlated Gaussian noise source. Section 3 contains the results of the commonly used Fokker-Planck approximation schemes $[14,15,20-22,27]$. Possible short-comings of those approximation schemes are pointed out. The activation rate is considered in Sect. 4. This activation rate is evaluated analytically by employing the perturbative Fokker-Planck schemes and is then compared with the exact results of a computer simulation. The conclusions are given in Sect. 5 .

\section{Bistable Stochastic Flow Driven by Colored Noise}

In what follows, we consider a stochastic flow of a one-dimensional order parameter $x(t)$. We assume a symmetric bistable flow modelled by the set of stochastic differential equations:

$\dot{x}=a x-b x^{3}+\xi \quad a, b>0$

$\dot{\xi}=-\frac{1}{\tau} \xi+\eta(t)$.

$\eta(t)$ is a stationary Gaussian white noise source of zero mean and correlation function

$\langle\eta(t) \eta(s)\rangle=\frac{2 D}{\tau^{2}} \delta(t-s)$.

Integration of $(2.1 \mathrm{~b})$ yields with $\xi\left(t_{0}=0\right)=\xi_{0}$

$\xi(t)=\xi_{0} \mathrm{e}^{-t / \tau}+\int_{0}^{t} \exp [-(t-s) / \tau] \eta(s) d s$

Assigning to the first two moments of $\xi_{0}$ the values given by the equations

$\left\langle\xi_{0}\right\rangle=0$

$\left\langle\xi_{0}^{2}\right\rangle=D / \tau$,

we find

$$
\langle\xi(t)\rangle=0
$$

and for the auto-correlation function the time-homogeneous result

$\langle\xi(t) \xi(s)\rangle=\frac{D}{\tau} \exp [-|t-s| / \tau]$.

Moreover, $\xi(t)$ is Gaussian and stationary only if prepared in Gaussian form consistent with (2.3a) and $(2.3 \mathrm{~b})$, i.e.

$\rho\left(\xi_{0}\right)=\left(\frac{\tau}{2 \pi D}\right)^{1 / 2} \exp \left[-\frac{\xi_{0}^{2}}{(2 D / \tau)}\right]$.
Thus, the system of differential equations in (2.1) is equivalent to a non-Markovian Langevin equation driven with additive Gaussian correlated noise

$\dot{x}=a x-b x^{3}+\xi(t)$,

with $\xi(t)$ obeying the properties in (2.4) and (2.5). Because $\langle\xi(t)\rangle=0$, the deterministic limit $(D \rightarrow 0)$ of (2.7) is clearly given by

$\dot{x}=a x-b x^{3} \equiv f(x)$

which is derivable from the potential $V(x)$

$V(x)=-\frac{a}{2} x^{2}+\frac{b}{4} x^{4}$

Furthermore, we will also implicitly assume that the Gaussian noise $\xi(t)$ in (2.7) is independent of the initial macroscopic random variable $x\left(t_{0}\right)=x_{0}$.

\section{Approximative Fokker-Planck Schemes}

It has been shown previously [5], that an exact closed form master equation for a nonlinear flow driven by colored noise $\xi(t)$ can in general not be written down explicitly. With $\xi(t)$ being a stationary Gaussian process obeying (2.3)-(2.6), the rate of change of the probability $p_{t}(x)$ obeys the formally exact relation $[5,14,15]$

$$
\begin{aligned}
\dot{p}_{t}(x)= & -\frac{\partial}{\partial x}\left(a x-b x^{3}\right) p_{t}(x) \\
& +\frac{D}{\tau} \frac{\partial^{2}}{\partial x^{2}} \int_{0}^{t} d s(\exp -(t-s) / \tau) \\
& \cdot\left\langle\frac{\delta x(t)}{\delta \xi(s)} \delta(x(t)-x)\right\rangle
\end{aligned}
$$

where $\delta x(t) / \delta \xi(s)$ denotes the functional derivative. This functional derivative obeys an exact integral equation [5] which for our case in (2.7) explicitly reads

$\frac{\delta x(t)}{\delta \xi(s)}=\theta(t-s) \cdot\left\{1+\int_{s}^{t} d \tau\left[a-3 b x^{2}(\tau)\right] \frac{\delta x(\tau)}{\delta \xi(s)}\right\}$

On expanding this relation into a Taylor series around $s^{-}=t$, and keeping only the first two terms, one obtains upon neglect of transients the so called short relaxation time Fokker-Planck approximation (SRTFPA)

SRTFPA: $\dot{p}_{t}(x)=-\frac{\partial}{\partial x}\left(a x-b x^{3}\right) p_{t}(x)$

$+D \frac{\partial^{2}}{\partial x^{2}}\left(1+\tau\left[a-3 b x^{2}\right]\right) p_{t}(x)$. 
Originally, (3.3) has been used by Stratonovich and Lax [27]. Recently this SRTFPA-scheme has been popularized by the authors of $[14,15]$. Furthermore, if one keeps within a formal Taylor series expansion of the functional derivative around $s^{-}=t[14,15]$ the terms proportional to $D \tau^{n}, n \geqq 0$, (and only those terms), one obtains the so-called "best Fokker-Planck approximation" (BFPA) introduced in $[15,22]$

$$
\text { BFPA: } \begin{aligned}
\dot{p}_{t}(x)= & -\frac{\partial}{\partial x}\left(a x-b x^{3}\right) p_{t}(x) \\
& +D \frac{\partial^{2}}{\partial x^{2}} H(x, \tau) p_{t}(x)
\end{aligned}
$$

where with $f(x)=a x-b x^{3}$, the "effective diffusion" $H(x, \tau)$ is given by [15] (prime denotes differentiation with respect to $x$ )

$$
\begin{aligned}
H(x, \tau)= & f(x)\left(1+\tau f(x) \frac{\partial}{\partial x}\right)^{-1} \frac{1}{f(x)} \\
= & f(x)\left(1-\tau f(x) \frac{\partial}{\partial x}\right. \\
+ & \left.\tau^{2} f(x) \frac{\partial}{\partial x} f(x) \frac{\partial}{\partial x}+\ldots\right) \frac{1}{f(x)} \\
= & 1+\tau f^{\prime}(x)+\tau^{2} \\
& \cdot\left[\left(f^{\prime}(x)\right)^{2}-f(x) f^{\prime \prime}(x)\right]+O\left(\tau^{3}\right) .
\end{aligned}
$$

At the zeros $\bar{x}=\left\{x_{1}, x_{u}, x_{2}\right\}$ of the deterministic flow (2.8) (extrema of $V(x)$ in (2.9)), one finds

$$
\begin{aligned}
H(\bar{x}, \tau) & =1+f^{\prime}(\bar{x}) \tau+\left(f^{\prime}(\bar{x})\right)^{2} \tau^{2}+\left(f^{\prime}(\bar{x})\right)^{3} \tau^{3}+\ldots \\
& =\left(1-f^{\prime}(\bar{x}) \tau\right)^{-1} .
\end{aligned}
$$

Thus, at the locally stable states $x_{1}, x_{2}, x_{1 / 2}$ $=\mp(a / b)^{1 / 2}$

$H\left(\bar{x}_{1}, \tau\right)=H\left(\bar{x}_{2}, \tau\right)=1 /(1+2 a \tau)$,

whereas at the locally unstable state $x_{u}$

$H\left(x_{u}, \tau\right)=1 /(1-a \tau)$.

A first difficulty with those Fokker-Planck approximation schemes emerges via the effective diffusion terms in (3.3) and (3.4), which are not necessarily positive for all $x$-values [15]; thereby generating generally unphysical boundaries. On the region of accessible $x$-values, as determined from (2.7), the probability $p_{t}(x)$ must stay positive (including zero) for all times $t$. Keeping the correlation time of the noise fixed, we by definition set the effective diffusion in $(3.3,3.4)$ zero within the region where it takes on negative values. The stationary probability $\bar{p}(x)$ of the corresponding Fokker-Planck approxi- mation scheme is then readily evaluated. For example, by use of (3.4) one obtains

$\bar{p}(x)=\frac{Z^{-1}}{H(x, \tau)}\left\{\exp +\frac{1}{D} \int_{0}^{x} \frac{f(y)}{H(y, \tau)} d y\right\} \theta(H(x, \tau))$

where the Heaviside step function $\theta(H(x, \tau))$ guarantees the positive support of $\bar{p}(x)$. Here and in the following we implicitly assume that the noise correlation time $\tau$ is small enough such that $\bar{p}(x)$ is nonvanishing within the bistable region $\left(x_{r}, x_{1}\right)$, where $x_{r}<x_{1}$ and $x_{l}>x_{2}, x_{1}<x_{2}$.

On the other hand, if one formally keeps all the terms generated by Taylor expansions of the functional derivatives $\delta x(t) / \delta \xi(s)$ around $s^{-}=t$, one obtains, upon neglect of all transients, for the rate of change of the probability $p_{t}(x)$ a Kramers-Moyal structure

$\dot{p}_{t}(x)=\sum_{n=1}^{\infty} \frac{(-1)^{n}}{n !}\left(\frac{\partial}{\partial x}\right)^{n}\left(K_{n}(x, \tau) p_{t}(x)\right)$.

Clearly, due to the neglect of transients, the master equations in $(3.3,3.4,3.10)$ can be utilized only for the evaluation of quantities determined by the asymptotic long time behavior of $p_{t}(x)$, such as the stationary probability or the leading behavior of a mean first pasage time (MFPT) at weak noise (Arrhenius factor). In this context, a recent paper [15] contains a somewhat confusing statement: In view of (3.10), (Eq. (2.28) in [15]) one reads: "Therefore, it is obvious that $K_{1}(x, \tau)$ and $K_{2}(x, \tau)$ contain only terms with coefficient $D \tau^{n}$ and in fact contain all such terms". From (3.2), however, we have for example for the second Taylor coefficient

$$
\begin{aligned}
\left.\frac{d^{2}}{d s^{2}} \frac{\delta x(t)}{\delta \xi(s)}\right|_{s^{-} \rightarrow t}= & \left\{\left(f^{\prime}(x(t))\right)^{2}-f(x(t)) f^{\prime \prime}(x(t))\right\} \\
& -f^{\prime \prime}(x(t)) \xi(t), \quad f^{\prime \prime}(x)=-6 b x
\end{aligned}
$$

If inserted into the small $\tau$-expansion of $\delta x(t) / \delta \xi(s)$, one obtains for the master operator in (3.10) the term

$$
\begin{aligned}
& D \tau^{2} \frac{\partial^{2}}{\partial x^{2}}\left\{\left[\left(f^{\prime}(x)\right)^{2}-f(x) f^{\prime \prime}(x)\right] p_{t}(x)+\frac{D}{\tau} f^{\prime \prime}(x) \frac{\partial}{\partial x} \int_{0}^{t}\right. \\
& \left.\cdot\left\langle\frac{\delta x(t)}{\delta \xi(s)} \delta(x(t)-x)\right\rangle \exp -\frac{(t-s)}{\tau} d s\right\} .
\end{aligned}
$$

Approximating the term $\delta x(t) / \delta \xi(s)$ occuring in (3.12) by one - see (3.2) - one finds from the last term in (3.12) a contribution

$D^{2} \tau^{2} \frac{\partial^{2}}{\partial x^{2}} f^{\prime \prime}(x) \frac{\partial}{\partial x} p_{t}(x)$. 
This term contributes obviously both to $K_{1}(x, \tau)$ and $K_{2}(x, \tau)$ in (3.10) if rearranged into Kramers-Moyal form. Thus, the BFPA-scheme does not contain all terms which contribute to the Fokker-Planck form; only if we simply neglect all terms, which also yield a contribution to a Kramers-Moyal moment $K_{n}(x, \tau)$ of order $n>2$, are $K_{1}(x, \tau)$ and $K_{2}(x, \tau)$ solely determined by the terms proportional to $D \tau^{n}$, yielding (3.4). More importantly, with a finite correlation time $\tau$, one obtains the term in (3.13), which has been neglected in $(3.3,3.4)$, but which is of the same order as the terms kept in $(3.3,3.4)$ (e.g. this follows directly from (3.13) by acting on $p_{t}(x)$ using the ansatz $\left.p_{t}(x, \tau) \propto \exp -\phi(x, \tau) / D\right)$. - The term in (3.11), as well as any other of the higher order Taylor coefficients occuring in the expansion of $\delta x(t) / \delta \xi(s)$ around the "Markovian time-point" $t$, generates a noise dependent term, and thus the same game starts over and over again. This results in an infinite hierarchy, each with infinite many series with terms proportional to $D^{m} \tau^{n}, m \leqq n, n>1$ (no analytic expansion). Therefore, it is a priori not clear to what extent the approximations in (3.3), (3.4) give a correct description of the tails of $\bar{p}(x)$, as determined by (3.10), when the correlation time of the noise source is non-vanishing.

The approximation in (3.9), which is based on (3.3) or (3.4), respectively, has been checked in special cases in [14] (transformations of Gaussian processes) and also in [15] and [21], by use of numerical and analogue simulations. For certain parameter regimes, those approximation schemes have provided satisfactory results for such quantities like stationary moments, location of maxima, etc. This very much resembles the case of a truncated Kramers-Moyal Fokker-Planck approximation to a Markovian master equation (integral operator), which often yields satisfactory results for similar quantities which are controled by the maximal weight of the probability $\bar{p}(x)$, despite the fact that $\bar{p}(x)$ is not exact. Based on the observation that all the Kramers-Moyal terms in (3.10) are generally of the same order, one would expect that an effective "best" Fokker-Planck approximation, modelling correctly the non-Markovian long-time behavior, must include also information about the higher order Kramers-Moyal moments in (3.10). Such a "renormalized" Fokker-Planck approximation scheme, modelling the long-time dynamics, has been put forward recently for the case of a Markovian master equation dynamics [28, 29].

A physical quantity, which sensitively probes the form of the stationary probability $\bar{p}(x)$, particularly in regions of small weight, is an activation rate. By use of a transport theory approach [12] to the master equation dynamics (3.10), the leading factor of the rate is solely determined by the ratio $\bar{p}\left(x_{u}\right) / \bar{p}\left(x_{1,2}\right)$ of the stationary probability $[12,29]$ (independently, this follows also from the asymptotic analysis of the MFPT of the underlying two-dimensional Fokker-Planck process in $(2.1)$ - see $[30,31]$ ); i.e. details of the boundary conditions reflect themselves only in prefactors of rate expressions. The study of this activation rate will be the subject of the next section.

\section{Activation Rates}

The activation rates of bistable flows present interesting physical quantities which depend crucially on the detailed form of the stationary probability $\bar{p}(x)$. Most naturally, one would like to evaluate the rate via a transport theory approach of the type used for dichotomic Markov noise [12]. In the absence of an exact master equation (3.10) modelling the long time behaviour of $x(t)$, this approach is of no use here. Alternatively, we could evaluate the MFPT at weak noise of the underlying two-dimensional Fokker-Planck dynamics in (2.1) $[30,31]$. However, because a detailed balance does not hold for (2.1), the standard methods [32-34] fail and the more general method of [30] and [31] is rather cumbersome, because the stationary probability $\bar{\rho}(x, \xi)$ must first be determined. If $T$ denotes the MFPT (mean first passage time) to reach the barrier top, the activation rate is estimated as

$r=1 / 2 T$

where the factor $1 / 2$ takes into account that the random walker has equal chance to either continue to the adjacent stable state or return to the old stable state.

The approximations in (3.3), (3.4) are of course meant to be useful small relaxation time approximations for the stationary dynamics. In what follows, we then look upon (3.3), (3.4) as a FokkerPlanck approximation to the long time dynamics of the (unknown) master equation dynamics (3.10). Then, within the assumption of a small enough relaxation time $\tau$, yielding a positive diffusion $\hat{D}(x)$ $=D H(x)$ within the bistable region $\left[x_{1}, x_{2}\right]$, the MFPT $T(x)$ can be readily evaluated $[35,36]$. If $x=$ $-\infty$ is a (natural) reflecting boundary and $x=x_{u}=0$ an absorbing state, one finds $[35,36]$ for the MFPT $T(x)$ of a walker which started out at $x(0)=x<0$

$T(x)=\int_{x}^{0} \frac{d y}{\bar{p}(y) \hat{D}(y)} \int_{-\infty}^{y} \bar{p}(z) d z$.

$\bar{p}(x)$ denotes the stationary probability of the corresponding Fokker-Planck equation (3.4) or (3.3) re- 
spectively, and $\hat{D}(x)$ is the corresponding diffusion coefficient, i.e. $\hat{D}(x)=D H(x)$ or $\hat{D}(X)=D(1+\tau(a-$ $\left.3 b x^{2}\right)$ ) if $(3.3)$ is utilized. For a weak noise, i.e. $D<a^{2} / b$ we can evaluate (4.2) by use of the method of steepest descendent. With the BFPA, (3.4), one obtains

$T=\frac{\pi}{a \sqrt{2}}\left(\frac{1+a \tau}{1-2 a \tau}\right)^{1 / 2} \exp (\Delta \phi / D)$

where with (3.5)

$$
\begin{aligned}
\Delta \phi= & \int_{0}^{x_{1}} \frac{f(y) d y}{H(y)}=\int_{0}^{-(a / b)^{1 / 2}} f(y)\left[1-\tau f^{\prime}(y)\right. \\
& \left.+\tau^{2} f(y) f^{\prime \prime}(y)+O\left(\tau^{3}\right)\right] d y \\
= & \frac{a^{2}}{4 b}\left(1-a^{2} \tau^{2}\right)+O\left(\tau^{3}\right) .
\end{aligned}
$$

Most importantly, the term linear in $\tau$ vanishes exactly. By use of (3.3) one finds instead

$T=\frac{\pi}{a \sqrt{2}}\left(\frac{1+2 a \tau}{1-a \tau}\right)^{1 / 2} \exp (\Delta \phi / D)$

with

$$
\begin{aligned}
\Delta \phi & =\int_{0}^{x_{1}} \frac{f(y) d y}{1+\tau f(y)} \\
& =\frac{a^{2}}{4 b}\left(1-a^{2} \tau^{2} / 2\right)+O\left(\tau^{3}\right) .
\end{aligned}
$$

Because (3.3) takes into account only the term of order $D \tau$, the term of order $\tau^{2}$ in $(4.4 \mathrm{~b})$ is, of course, meaningless. Again, the term of order $\tau$ in $\Delta \phi$ vanishes. For $\tau=0$ both results (4.3) and (4.4) coincide and $r=1 / 2 T$ equals the well-known Smoluchowski rate $[35,36]$.

Most importantly, we note that the Arrhenius factor of $T(x)$

$\exp (\Delta \phi / D)$

does not exhibit a correlation time dependence in first order in $\tau$ ! Based on the SRTFPA in (3.3), the advocates of Ref. 15 construct an approximation for the stationary probability of (3.3) of the form [15, $16]$

$\bar{p}(x)=p_{0}(x)+\tau p_{1}(\tau)+O\left(\tau^{2}\right)$

where $p_{0}(x)$ is the white noise stationary probability $p_{0}(x)$, which in our case reads

$p_{0}(x)=Z^{-1} \exp \left(-\frac{-a x^{2} / 2+b x^{4} / 4}{D}\right)$.

By use of their result in (2.23) of [15], one obtains in our case (2.7)

$$
\begin{aligned}
\bar{p}(x)= & p_{0}(x)\left\{1+\tau\left[C-\left(a-3 b x^{2}\right)\right.\right. \\
& \left.\left.-\frac{1}{2 D}\left(a x-b x^{3}\right)^{2}\right]\right\}
\end{aligned}
$$

where

$$
C=-\frac{1}{2 D} \int_{-\infty}^{+\infty}\left(a x-b x^{3}\right)^{2} p_{0}(x) d x<0 .
$$

This approximation for $\bar{p}(x)$ is not necessarily positive. Considering (4.8) as a short-time approximation in first order, the bracket in (4.8) is now exponentiated [15]. This ad hoc [15] exponentiation guarantees a positive $\bar{p}(x)$, which explicitly reads

$$
\bar{p}(x)=p_{0}(x)
$$

$\cdot \exp \left(-\frac{D \tau\left[|C|+\left(a-3 b x^{2}\right)+\frac{1}{2 D}\left(a x-b x^{3}\right)^{2}\right]}{D}\right)$.

If we take (4.10) seriously, we would obtain a relevant factor of $T$ given by

$$
\begin{aligned}
\frac{\bar{p}\left(x_{1}\right)}{\bar{p}(x=0)} & =\exp \frac{a^{2} / 4 b+3 D \tau a}{D} \\
& =\exp (3 \tau a) \cdot \exp \left(\frac{a^{2} / 4 b}{D}\right) .
\end{aligned}
$$

In this case, the relevant factor does exhibit a dependence on $\tau$, but in the form of a mere prefactor correction, $\exp (3 a \tau)$. The results in (4.3), (4.4) and (4.11) are in clear contrast to a result found for symmetric dichotomic noise where the Arrhenius factor increases with increasing correlation time $\tau$ [12]. In view of the absence of a term proportional to $\tau$ in the Arrhenius factors (4.3), (4.4), (4.11), we performed a numerical simulation for $T$ based on the bistable flow (2.7). The results are given in Fig. 1. In contrast to our forecastings in (4.3), (4.4) and (4.11) $\Delta \phi$ is increasing with increasing correlation time $\tau$. The increase is proportional to first order in $\tau$ and is not really dependent on the small noise parameter $D$. These results imply the following conclusions:

(i) Because the calculated Arrhenius factors disagree with the simulation, the Fokker-Planck approximation schemes in (3.3), (3.4) cannot be correct in leading order in $\tau$ if viewed as a long time approximation to the master equation dynamics (3.10). In other words, the stationary probability $\bar{p}(x),(3.9)$, of (3.4) cannot be equal to the exact stationary proba- 


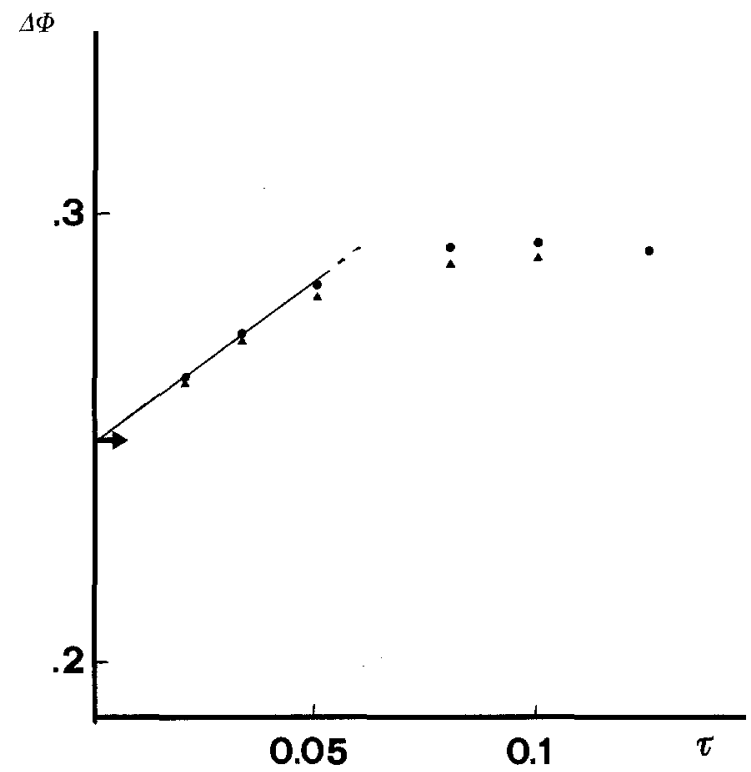

Fig. 1. $\Delta \phi$, defined in (4.5), versus the noise auto-correlation time $\tau$. The computer simulation of (2.1) has been carried out by applying the numerical algorithm of Ref. 15 with an integration step of 0.01 . The values of the parameters are $a=b=1, D=0.1(\bullet)$ and $D=0.05(\mathbf{\Delta}) . T$ is the average over 1,000 first passage times occurred from the initial conditions (2.6) for $\xi_{0}$ and $p(x, 0)=$ $\delta\left(x-x_{1}\right)$ for $x$. The maximum error bar in our numerical simulation is estimated to be about $10 \%$. The arrow denotes the white noise limit, $\Delta \phi(\tau=0)$

bility $\bar{p}(x)$ in leading order in $\tau$ as determined from (2.7) or, equivalently, from the (unknown) master equation dynamics (3.10).

(ii) The approximation in (4.11) based on the ad hoc exponentiation scheme of (4.6) can also not be correct in leading order in $\tau$ if compared with the exact probability. This fact is not remedied if (4.6) is used in connection with (3.4) instead of (3.3); the correlation time $\tau$ is merely substituted by a "renormalized" correlation time $\tau_{R}[15]$.

\section{Discussion and Conclusions}

We presented a study of a simple nonlinear bistable flow which is driven by exponentially correlated, additive Gaussian noise and evaluated the leading behavior of the activation rate at low noise. This leading term of the activation rate is determined by the leading order of the ratio of the stationary probability, taken at the locally unstable state $x=x_{u}$ and the metastable state $x=x_{1}$ (or $x_{2}$ ). Our numerical results for this leading term indicate that the commonly used Fokker-Planck approximation schemes $[14,15,22,27]$ yield a stationary probability $\bar{p}(x, \tau)$ in which the tails are not recovered correctly. This important short-coming should not come as too big a surprise. The exact relation in (3.1) inherits via the functional derivative $\delta x(t) / \delta \xi(s)$ a rather complex structure. This functional derivative embodies the complex nonlinear dependence of $x(t)$ on the previous noise history $\left\{\xi(s), t_{0} \leqq s \leqq t\right\}$. This complexity becomes exposed immediately if one attempts to expand this nonlinear functional around the "Markovian" time-point $s^{-}=t$ (see Sect. 3), which in turn yields a never ending infinite hierarchy of infinite many contributions. This is the price being paid by having reduced the underlying two-dimensional phase - space dynamics $(x(t), \xi(t)),(2.1)$, onto a onedimensional, but non-Markovian dynamics. In particular, it should also be kept in mind, that for a nonlinear, nonequilibrium dynamics of the type in (2.1), even the asymptotic dynamics at a low noise level is almost never integrable [30b, 37].

All of that clearly causes headaches for the theorists; - an "a priori" use of those perturbative (truncated) Fokker-Planck schemes $[14,15,22,27]$, together with related perturbative methods for the probability itself $[15,16,38]$ is generally somewhat suspect. Those cases, for which the effective Fokker-Planck structure becomes exact, can be related to nonlinear (eventually also time-dependent) transformations of non-Markovian Gaussian processes $[5,14,19]$. In general, the master equation has the structure of an integral operator, (3.10), and a Fokker-Planck approximation to the long-time behavior should then also include information about the integral operator structure $[28,29]$ if the global behavior of stationary probabilities is to be described accurately.

Part of the above reasoning equally well applies to the numerous papers written on the subject of systematic adiabatic elimination procedures such as e.g. the study of corrections to the Smoluchowski equation and alike [39]. The bulk of those papers implicitly contain some of the same assumptions inherent in the derivation of the approximative Fokker-Planck schemes in (3.3), (3.4) $[14,15,22,26,27$, 39]. Furthermore, related there is also the problem of correct initial values; i.e. initial preparation effects $[23 \mathrm{~b}, 25,40,41]$. Different initial preparation schemes (for the details see in Refs. 23b, 25) give rise to different (memory-dependent or time-convolutionless) master equations propagating the single-event probability $p_{t}[5,25]$, and related, different nonMarkovian Langevin equations [25]. It should be noted, that the non-white noise properties, such as the conditional average $\left\langle\xi(t) \mid x\left(t_{0}\right)=x\right\rangle$ (or also initial correlations $\left.\left\langle\xi\left(t_{0}\right) x\left(t_{0}\right)\right\rangle\right)$ depend on the initial preparation scheme. The preparation scheme consistent with (3.1) refers to a "correlation-free" initial preparation; i.e. the initial probability $\rho^{T}\left(\xi_{0}, x\right)$ of the total system factorizes

$\rho^{T}\left(\xi_{0}, x\right)=\rho\left(\xi_{0}\right) p_{0}(x)$

where $\rho\left(\xi_{0}\right)$ denotes the Gaussian in (2.6) and $p_{0}(x)$ 
denotes the (otherwise arbitrary) initial macroprobability. Also, the dependence of the stochastic kernels on the initial preparation $[5,25]$ does in general not die out (because the initial preparation, determining e.g. the projector operator kernels, is not being propagated in time); nevertheless, with an ergodic behavior [1], the single-event probability $p_{t}$ approaches the stationary probability, $\lim _{t \rightarrow \infty} p_{t}=\bar{p}$, which, of course, is not dependent on the initial preparation scheme. $\bar{p}(x)$ just represents the zero-mode of generally preparation dependent [25] limiting $(\operatorname{Lim} t \rightarrow \infty)$ master operators of the type in (3.10), where the transient terms have died out. Therefore, if the noise source has a nonvanishing correlation time $\tau$, the limiting (non-Markovian)master operators of the type in (3.3), (3.4) and (3.10) yield information about quantities which involve zero-modes only; e.g. the stationary probability $\bar{p}(x)$, or current carrying stationary non-equilibrium probabilities [9-12], which clearly also do not depend on the initial preparation.

\section{References}

1. Hänggi, P., Thomas, H.: Phys. Rep. 88 C, 209 (1982)

2. Risken, H.: The Fokker-Planck equation: methods of solution and applications. In: Springer Series in Synergetics. Haken, $H$. (ed.). Berlin, Heidelberg, New York: Springer 1983

3. Mori, H.: Prog. Theor. Phys. 34, 399 (1965)

4. Kubo, R.: Rep. Prog. Phys. 29, 255 (1966)

5. Hänggi, P.: Z. Phys. B - Condensed Matter 31, 407 (1978); Hänggi, P., Talkner, P.: Phys. Lett. 68 A, 9 (1978); Hänggi, P.: Phys. Lett. 83 A, 196 (1981)

6. Grigolini, P.: J. Stat. Phys. 27, 283 (1982)

7. Kubo, R.: In: Fluctuations, relaxation and resonance in magnetic systems. ter Haar, Oliver, Boyd (eds.), p. 23 ff. Edinburgh 1962

8. Grote, R.F., Hynes, J.T.: J. Chem. Phys. 73, 2715 (1980)

9. Hänggi, P., Mojtabai, F.: Phys. Rev. A 26, (Rap. Comm.) 1168 (1982); J. Stat. Phys. 30, 401 (1983)

10. Marchesoni, F., Grigolini, P., Marin, P.: Chem. Phys. Lett. 87, 541 (1982); Marchesoni, F., Grigolini, P.: J. Chem. Phys. 78, 6287 (1983); Hänggi, P., Weiss, U.: Phys. Rev. A 29, 2265 (1984)

11. Carmeli, B., Nitzan, A.: Phys. Rev. A 29, 1481 (1984)

12. Hänggi, P., Riseborough, P.: Phys. Rev. A 27, (Rap. Comm.), 3379 (1983); Broeck, C. Van den, Hänggi, P.; Phys. Rev. A 30 (in press)

13. Kitahara, K., Horsthemke, W., Lefever, R., I. Inaba: Prog. Theor. Phys. 64, 1233 (1980)

14. Sancho, J.M., San Miguel, M.: Z. Phys. B - Condensed Matter 36, 357 (1980); 43, 361 (1981)

15. Sancho, J.M., San Miguel, M., Katz, S.L., Gunton, J.D.: Phys. Rev. A 26, 1589 (1982)

16. Horsthemke, W., Lefever, R.: Z. Phys. B - Condensed Matter 40, $241(1980)$

17. Marchesoni, F., Grigolini, P.: Physica A 121, 269 (1983)

18. Horsthemke, W., Lefever, R.: Noise induced transitions: theory and applications in physics, chemistry and biology. In: Springer Series in Synergetics. Berlin, Heidelberg, New York, Tokyo: Springer 1984

19. Hänggi, P.: Z. Phys. B - Condensed Matter 30, 85 (1978)

20. Garrido, L., Sancho, J.M.: Physica A 115, 479 (1982)
21. Sancho, J.M., San Miguel, M., Yamazaki, H., Okada, T.: Physica A 116, 560 (1982)

22. Kampen, N.G. Van: Phys. Rep. 24C, 171 (1976); pp. $210 \mathrm{ff}$.

23. Hänggi, P., Thomas, H., Grabert, H., Talkner, P.: J. Stat. Phys. 18, 155 (1978); Z. Phys. B - Condensed Matter 29, 273 (1978)

24. Hänggi, P., Thomas, H.: Z. Phys. B - Condensed Matter 26, 85 (1977)

25. Grabert, H., Hänggi, P., Talkner, P.: Z. Phys. B - Condensed Matter 26, 389 (1977); J. Stat. Phys. 22, 537 (1980)

26. Hernandez-Machado, A., Sancho, J.M., San Miguel, M., Pesquera, L. Z. Phys. B - Condensed Matter 52, 353 (1983)

27. Stratonovich, R.L.: Topics in the theory of random noise. Vol. I, p. 98. New York: Gordon and Breach 1963; Lax, M.: Rev. Mod. Phys. 38, 541 (1966)

28. Hänggi, P.: Phys. Rev. A 25, 1130 (1982); Grabert, H., Hänggi, P., Oppenheim, I.: Physica A 119, 300 (1983)

29. Hänggi, P., Grabert, H., Talkner, P., Thomas, H.: Phys. Rev. A 29, 371 (1984)

30. Talkner, P., Hänggi, P.: Phys. Rev. A 29, 768 (1984); with Ryter, D.: In: Proc. on noise in physical systems and $1 / f$ noise. Savelli, M., et al. (eds.), p. 63. New York: Elsevier Science Publ, 1983

31. Matkowsky, B.J., Schuss, Z.: SIAM J. Appl. Math. 33, 365 (1977); Schuss, Z.: SIAM Rev. 22, 119 (1980)

32. Kramers, H.A.: Physica 7, 284 (1940)

33. Landauer, R., Swanson, J.A.: Phys. Rev. 121, 1668 (1961)

34. Langer, J.S.: Ann. Phys. (NY) 54, 258 (1969)

35. Stratonovich, R.L.: Topics in the theory of random noise. Vol. I, pp. 79-83. New York: Gordon and Breach 1963

36. Schulten, K., Schulten, Z., Szabo, A.: J. Chem. Phys. 74, 4426 (1981)

37. Graham, R., Tel, T.: Preprint 1984

38. Blankenship, C., Papanicolaou, G.C.: SIAM J. Appl. Math. 34, 437 (1978)

39. As a representative sample of papers on this subject we list: Wilemski, G.: J. Stat. Phys. 14, 153 (1976); Titulaer, U.M.: Physica A 91, 321 (1978); A 100, 251 (1980); Skinner, J.L., Wolynes, P.: Physica A 96, 561 (1979); Chartuverdi, S., Shibata, F.: Z. Phys. B - Condensed Matter 35, 297 (1979); Wodkiewicz, K.: J. Math. Phys. 20, 46 (1979); Risken, H., Vollmer, H.D., Morsch, M.: Z. Phys. B - Condensed Matter 40, 343 (1980); San Miguel, M., Sancho, J.M.: J. Stat. Phys. 22, 605 (1980); Schenzle, A., Graham, R.: Phys. Rev. A 26, 1976 (1982); Phys. Lett. 98 A, 319 (1983); Marchesoni, F., Grigoloini, P.: Physica A 121, 269 (1983)

40. Haake, F., Lewenstein, M.: Phys. Rev. A 28, 3606 (1983)

41. Marchesoni, F.: Phys. Lett. 101 A, 11 (1984)

P. Hänggi

Department of Physics

Polytechnic Institute of New York

333 Jay Street

Brooklyn, NY 11201

USA

F. Marchesoni

Dipartmento di Fisica

Università di Perugia

Via Elce di Sotto

I-06100 Perugia

Italy

P. Grigolini

Dipartmento di Fisica

and GNSM-CNR

P. za Torricelli 2

I-56100 Pisa

Italy 